\title{
Isomerization in Alkynyl-Protected Gold Nanoclusters
}

\author{
Zong-Jie Guan ${ }^{\dagger, \ddagger}$, Feng $\mathrm{Hu}^{\dagger}$, Jiao-Jiao Li ${ }^{\dagger}$, Zhao-Rui Wen ${ }^{\ddagger}$, Yu-Mei Lin ${ }^{\ddagger}$ and Quan-Ming Wang*,†, \\ ${ }^{\dagger}$ Department of Chemistry, Tsinghua University, Beijing, 100084, P. R. China. \\ tDepartment of Chemistry, College of Chemistry and Chemical Engineering, Xiamen University, Xiamen, 361005, P. R. \\ China.
}

\section{Table of Contents}

\section{Physical measurements}

\section{Computational details.}

\section{Supporting Figures}

Figure S1. Binding motifs in $\mathbf{A u}_{23}-\mathbf{1}, \mathbf{A u}_{23}-\mathbf{2}$.

Figure S2. The electronic diagrams of HOMO-1, HOMO, LUMO and LUMO+1 of a) $\mathbf{A u}_{23-1} \mathbf{1}$ and b) $\mathbf{A u}_{\mathbf{2 3}} \mathbf{- 2}$.

Figure S3. Experimental and calculated absorption spectra using ADF 2017 of a) $\mathbf{A u}_{23} \mathbf{- 1}$ and b) $\mathbf{A u}_{23} \mathbf{- 2}$.

Figure S4. Kohn-Sham molecular orbital energy levels diagram and the associated populations of atomic orbitals in each KS molecular orbital for both $\mathrm{Au}_{23}$ clusters.

Figure S5. The electronic density diagrams of the $\mathrm{Au}_{15}{ }^{7+}$ kernel in two $\mathrm{Au}_{23}$ clusters with superatom-like $\mathrm{P}$ orbital characters.

Figure S6. Photographs of the $\mathbf{A u}_{23}-\mathbf{2}$ before and after the transformation in $\mathrm{CH}_{2} \mathrm{Cl}_{2}$.

Figure S7. MALDI-TOF mass spectra of the product formed by cluster-conversion reaction of $\mathbf{A u}_{\mathbf{2 3}} \mathbf{- 2}$.

Figure S8. Time-course NMR spectra of cluster-conversion from $\mathbf{A u}_{23}-\mathbf{2}$ to $\mathbf{A u}_{23}-\mathbf{1}$ in $\mathrm{CD}_{2} \mathrm{Cl}_{2}$.

Figure S9. Time-dependent UV-vis absorption spectra of $\mathbf{A u}_{23}-\mathbf{2}$ under different conditions.

Figure S10. The diagram of transformation process from $\mathbf{A u}_{23}-\mathbf{2}$ to $\mathbf{A u}_{23}-\mathbf{1}$.

Figure S11. The optical absorption spectra of two $\mathrm{Au}_{23}$ isomers (photon-energy plot).

Figure S12. The relative energy difference between $\mathbf{A u}_{23} \mathbf{- 1}$ and $\mathbf{A} \mathbf{u}_{23}-\mathbf{2}$ calculated by Gaussian 09 .

Table S1. Selected Au-Au bond lengths ( $\AA$ ) for the Experimental and b3lyp/genecp (6-31G* for C, H and lanl2dz for $\mathrm{Au}$ ) optimized structure of $\mathbf{A u}_{\mathbf{2 3}} \mathbf{- 1}$.

Table S2. Selected Au-Au bond lengths ( $\AA$ ) for the Experimental and b3lyp/genecp (6-31G* for C, H and lanl2dz for $\mathrm{Au}$ ) optimized structure of $\mathbf{A} \mathbf{u}_{23} \mathbf{- 2}$.

Table S3. The transitions corresponding to the significant peaks of $\mathbf{A} \mathbf{u}_{23}-\mathbf{1}$ and $\mathbf{A} \mathbf{u}_{23}-\mathbf{2}$ cluster calculated according to TDDFT method using the quantum chemistry program Gaussian 09 .

Table S4. Frontier molecular orbital compositions (\%) in the ground state for two $\mathrm{Au}_{23}$ isomers at the CAMB3LYP/6-31G* \& LANL2DZ level.

Table S5. The transitions corresponding to the significant peaks of $\mathbf{A u}_{23}-\mathbf{1}$ and $\mathbf{A} \mathbf{u}_{23}-\mathbf{2}$ cluster calculated according to TDDFT method using Amsterdam Density Functional (ADF 2017) software package.

Table S6. Crystal data and structure refinement for $\mathbf{A u}_{23} \mathbf{- 1}$ and $\mathbf{A} \mathbf{u}_{23}-\mathbf{2}$. 


\section{Physical measurements}

Characterization. UV-VIS-NIR absorption spectra were recorded on cary5000. Mass spectra were recorded on Bruker MicroFlex MALDI-TOF-MS and Shimadzu AXIMA Performance-MALDI-TOF-MS. Nuclear magnetic resonance (NMR) data were recorded on a JEOL ECS-400 spectrometer (400 MHz). Intensity data of compounds $\mathbf{A u}_{23}-\mathbf{1}$ and $\mathbf{A u}_{23}-\mathbf{2}$ were collected on a Rigaku Agilent SuperNova Dual system (Mo K) at $100 \mathrm{~K}$. Absorption corrections were applied by using the program CrysAlis (multi-scan). The structure was solved by direct methods, and non-hydrogen atoms were refined anisotropically by least-squares on $F^{2}$ using the SHELXTL program. The hydrogen atoms of organic ligands were generated geometrically. SQUEEZE tool of PLATON was applied to both $\mathbf{A u}_{23}-\mathbf{1}$ and $\mathbf{A} \mathbf{u}_{23}-\mathbf{2}$, due to large solvent voids in the structures.

Method for determining optical gaps. In the plotting of absorbance $(A)$ against photon energy $(h v)$ (Figure S11), ${ }^{1-3}$ a tangent line is drawn at the inflection point on lower energy absorption band, and the line is extended all the way to the x-axis. By extrapolating the absorbance to zero, the optical bandgaps of $\mathbf{A u}_{\mathbf{2 3}} \mathbf{- 1}$ and $\mathbf{A} \mathbf{u}_{\mathbf{2 3}} \mathbf{- 2}$ are determined to be 1.847 and $1.797 \mathrm{eV}$, respectively.

Experimental procedure for cluster transformation from $\mathbf{A u}_{23}-\mathbf{2}$ to $\mathbf{A u}_{23}-1$ : The sample of $\mathbf{A u}_{23}-\mathbf{2}$ was dissolved in the organic solvents and treated by the following procedures and real-time monitored by UV-Vis spectrometry. (A) The sample was dissolved in $2 \mathrm{~mL}$ of dichloromethane, $25^{\circ} \mathrm{C}$, in the presence of $\mathrm{O}_{2}$ (Figure 8); (B) The sample was dissolved in $2 \mathrm{~mL}$ of dichloromethane, $4^{\circ} \mathrm{C}$, in the presence of $\mathrm{O}_{2}$ (Figure S9a). (C) The sample was dissolved in 2 $\mathrm{mL}$ of dichloromethane, $25^{\circ} \mathrm{C}$, in the presence of $\mathrm{O}_{2}$, Addition of 1 equiv $\mathrm{Ph}_{4} \mathrm{PCl}$ (Figure $\mathrm{S} 9 \mathrm{~b}$ ).

\section{Computational details}

Time-dependent DFT calculations of the UV-vis absorption were performed with the quantum chemistry program Gaussian $09^{4}$ and ADF $2017.5^{5}$

In Gaussian calculations, the 6-31G(d) basis set is used for $\mathrm{C}$ and $\mathrm{H}$, and LANL2DZ for Au. ${ }^{6-7}$ Geometry optimizations were done with the functional of B3LYP. Time-dependent DFT calculations were done with the hybrid functionals of CAM-B3LYP. ${ }^{8}$ Sixty singlet states are chosen in the calculations of the UV-vis absorption spectra for two clusters. All transitions together with their oscillator strengths were then convoluted with a Gaussian line shape of $0.4 \mathrm{eV}$ broadening to make the whole optical-absorption spectrum.

In ADF calculations, time-dependent density functional theory calculations using the SAOP potential. ${ }^{9}$ DZP basis sets were used for $\mathrm{C}, \mathrm{H}$ atoms, and TZP basis sets were used for Au atoms. Scalar relativistic effects were incorporated by utilizing the zeroth-order regular approximation. Fifty singlet states are chosen in the calculations of the UV-vis absorption spectra. All transitions together with their oscillator strengths were then convoluted with a Gaussian line shape of $0.13 \mathrm{eV}$ broadening to make the whole optical-absorption spectrum.

The theoretical results calculated by Gaussian are compared with experimental ones without any transforming or post-processing (spectra shifted or stretched). In ADF calculations, TDDFT spectra were post-processed to facilitate comparison to experimental ones, for instance, the calculated spectra of $\mathbf{A} \mathbf{u}_{23}-\mathbf{1}$ and $\mathbf{A u}_{\mathbf{2 3}} \mathbf{- 2}$ were shifted and stretched, respectively. Different scales are used for showing theoretical and experimental results in the UV spectra (Figure S3). 


\section{Supporting Figures.}
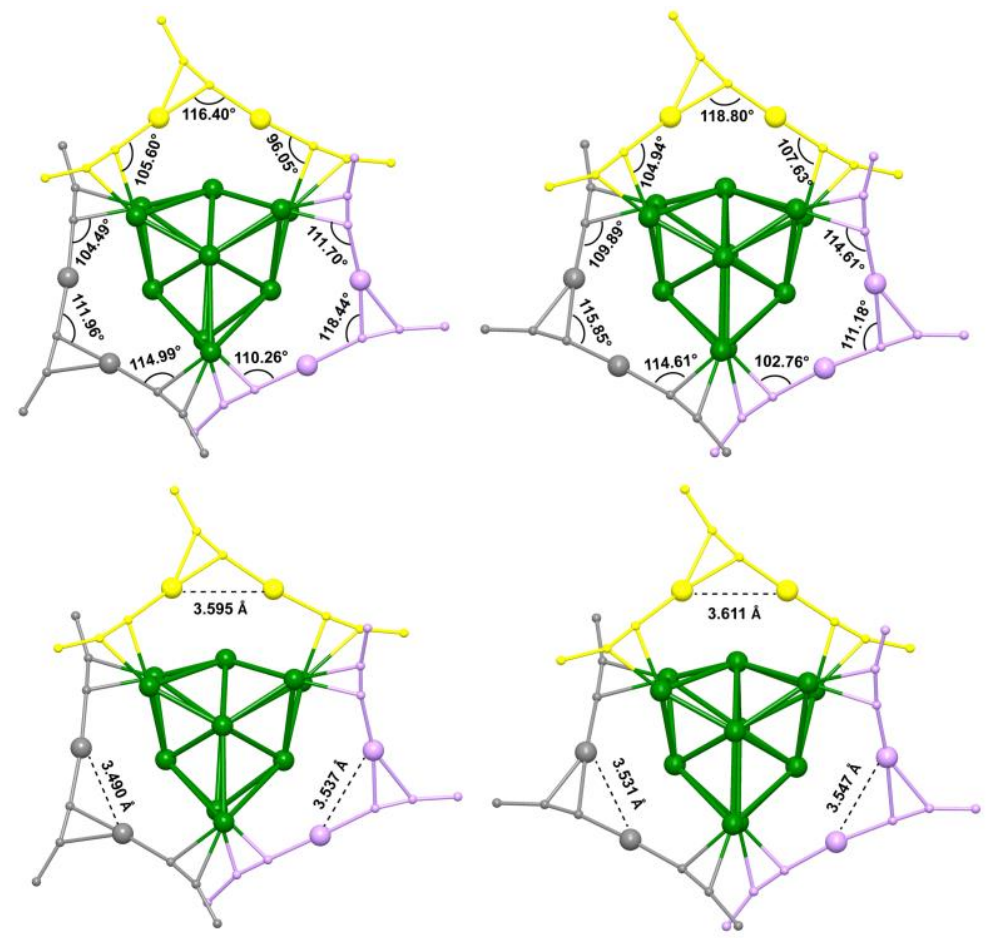

$\mathrm{Au}_{23}-1$

$\mathrm{Au}_{23}-2$

Figure S1. Binding motifs in $\mathbf{A u}_{23}-\mathbf{1}, \mathbf{A u}_{23}-\mathbf{2}$. Color codes: green, $\mathrm{Au}_{11}$ nuit; yellow, gray, lavendar, V-shaped alkynyl-gold staple motif. 
a)
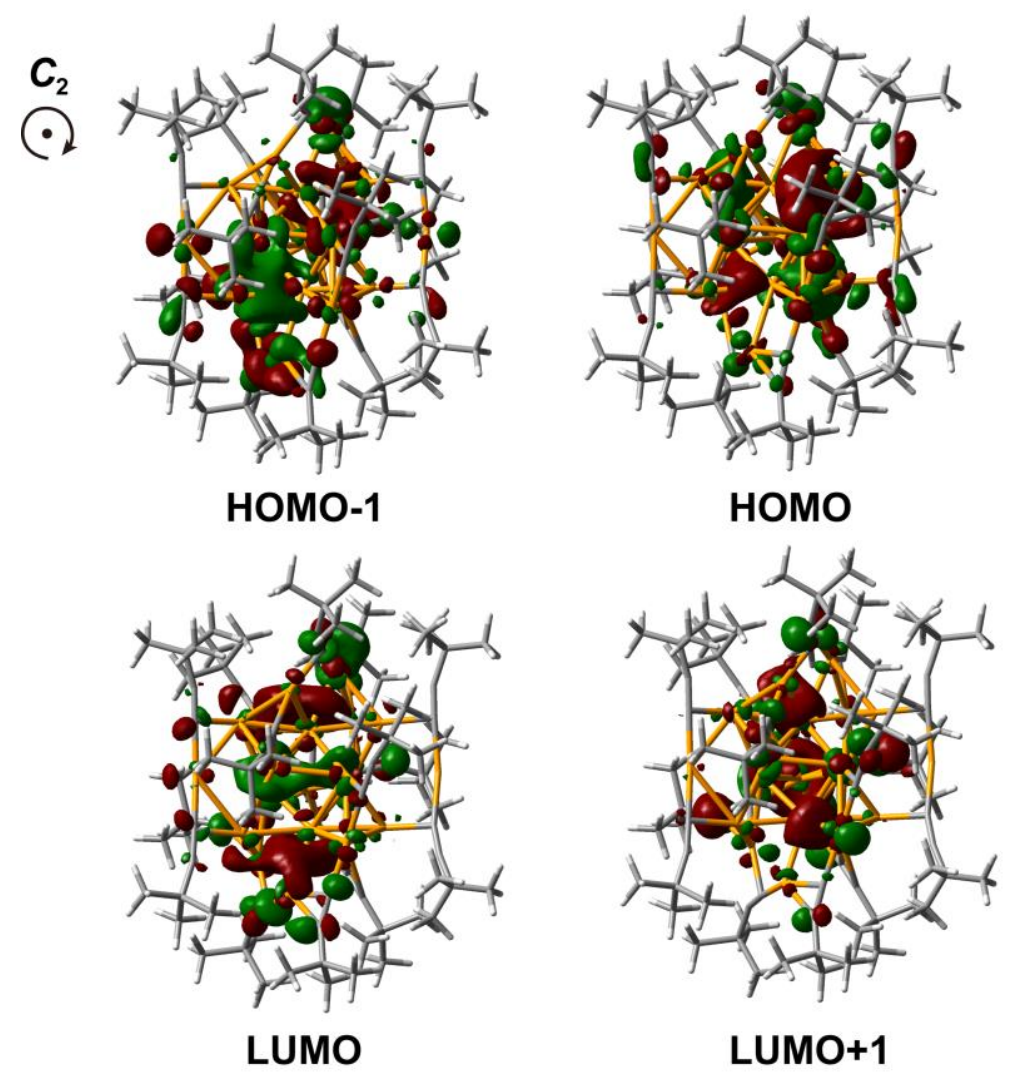

b)

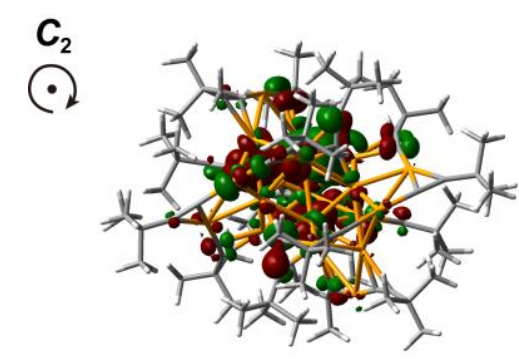

HOMO-1

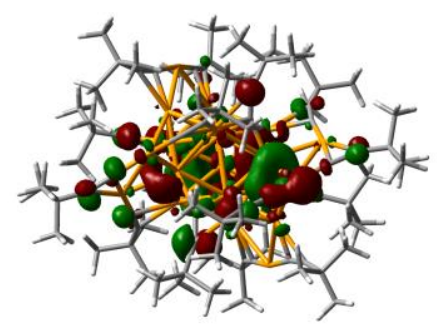

LUMO

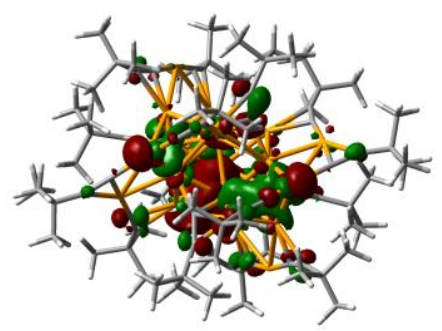

HOMO

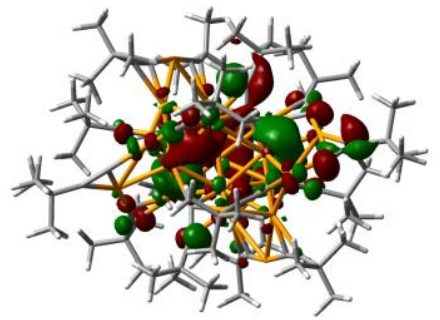

LUMO+1

Figure S2. The electronic diagrams of HOMO-1, HOMO, LUMO and LUMO+1 of a) $\mathbf{A} \mathbf{u}_{23} \mathbf{- 1}$ and b) $\mathbf{A u}_{\mathbf{2 3}} \mathbf{- 2}$. 

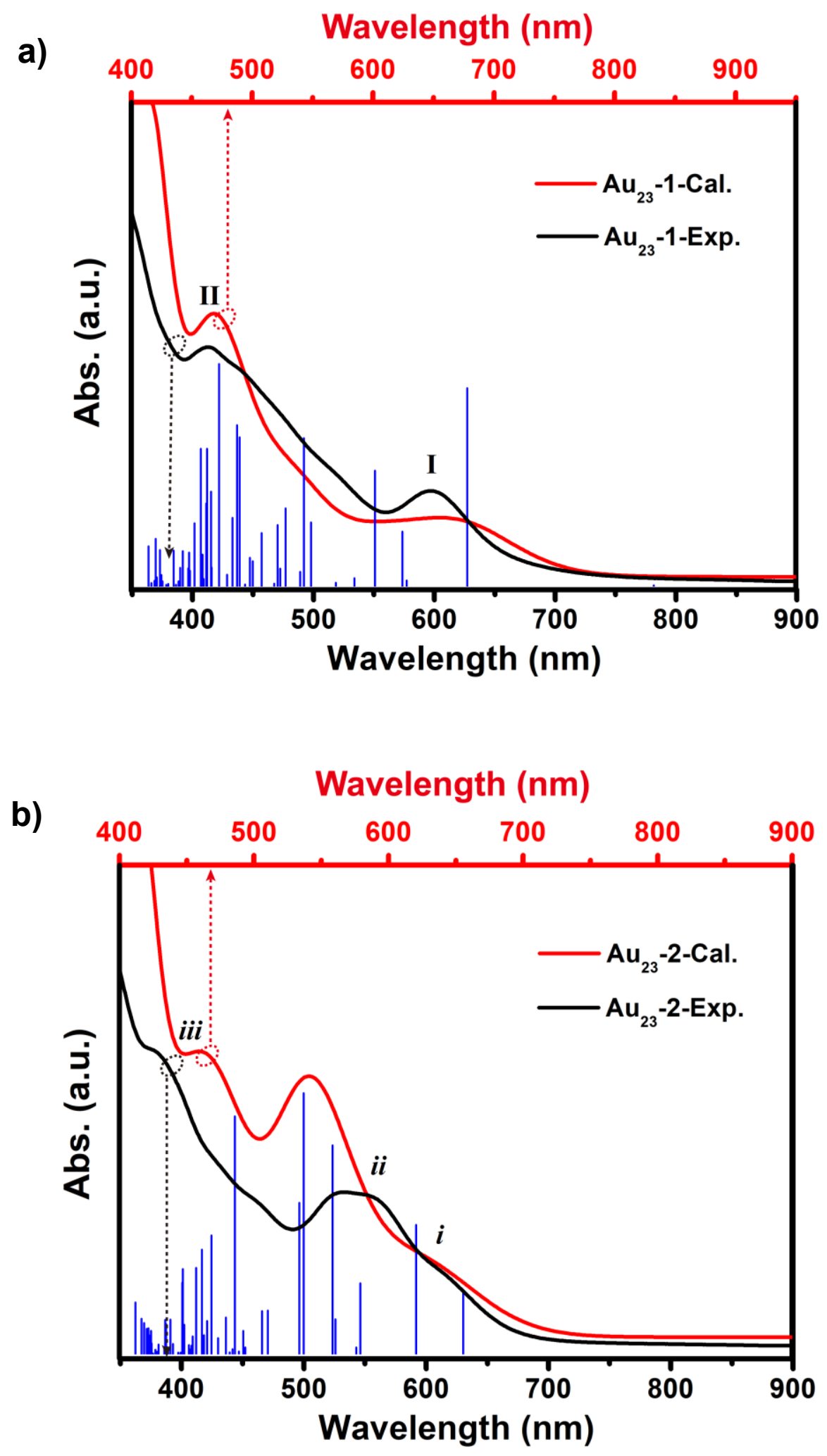

Figure S3. Experimental (black) and calculated (red) absorption spectra using ADF 2017 of a) $\mathbf{A u}_{23} \mathbf{- 1}$ and b) $\mathrm{Au}_{23}-2$. 

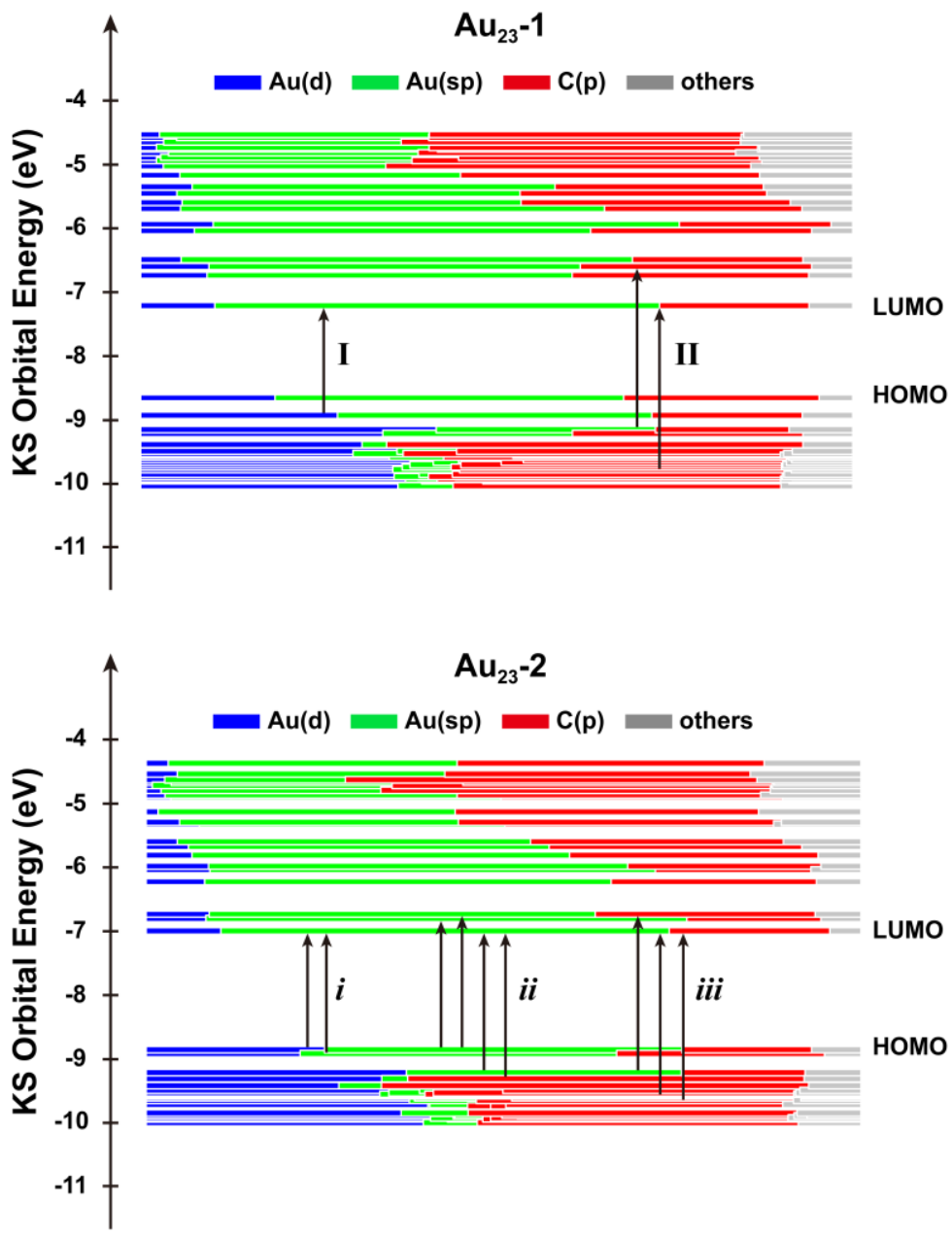

Figure S4. Kohn-Sham molecular orbital energy levels diagram and the associated populations of atomic orbitals in each $\mathrm{KS}$ molecular orbital for both $\mathrm{Au}_{23}$ clusters.

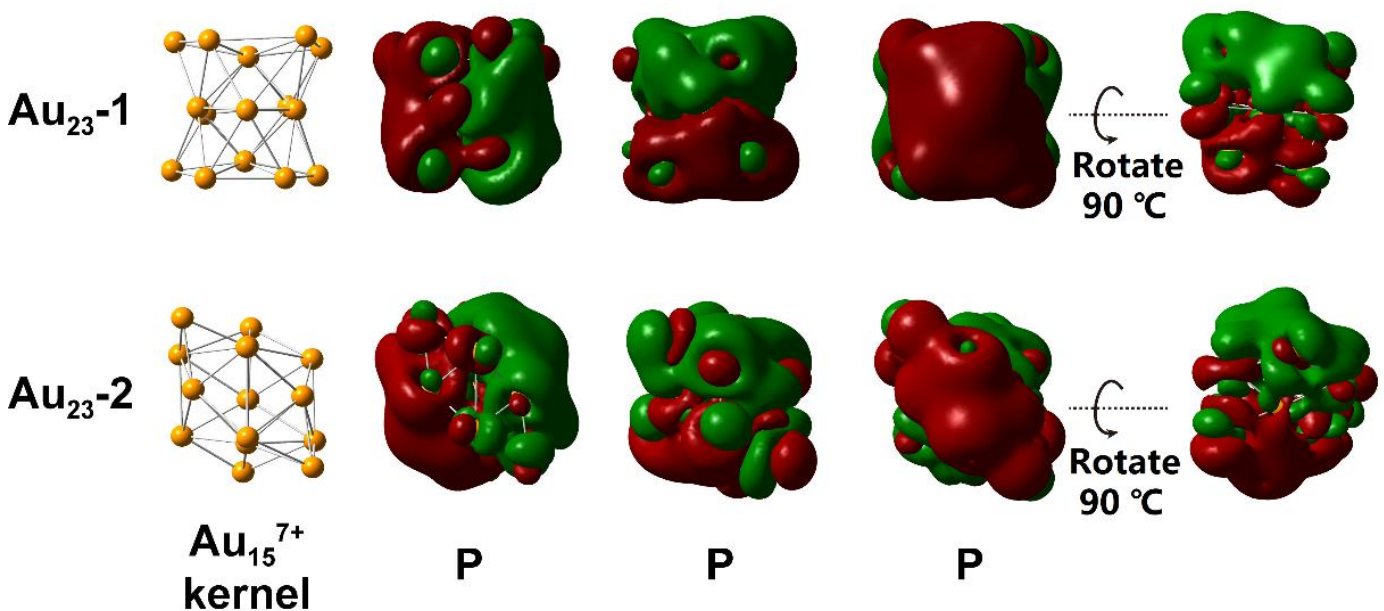

Figure S5. The electronic density diagrams of the $\mathrm{Au}_{15}{ }^{7+}$ kernel in two $\mathrm{Au}_{23}$ clusters with superatom-like $\mathrm{P}$ orbital characters. 


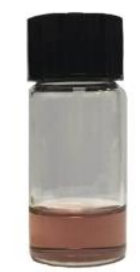

\section{After $7 \mathrm{~h}$}

\section{IIIII $\Rightarrow$ \\ In $\mathrm{CH}_{2} \mathrm{Cl}_{2}$}

$\mathrm{Au}_{23} \mathrm{-2}$

$\mathrm{Au}_{23}=1$

Figure S6. Photographs of the $\mathbf{A u}_{23}-\mathbf{2}$ before and after the transformation in $\mathrm{CH}_{2} \mathrm{Cl}_{2}$.

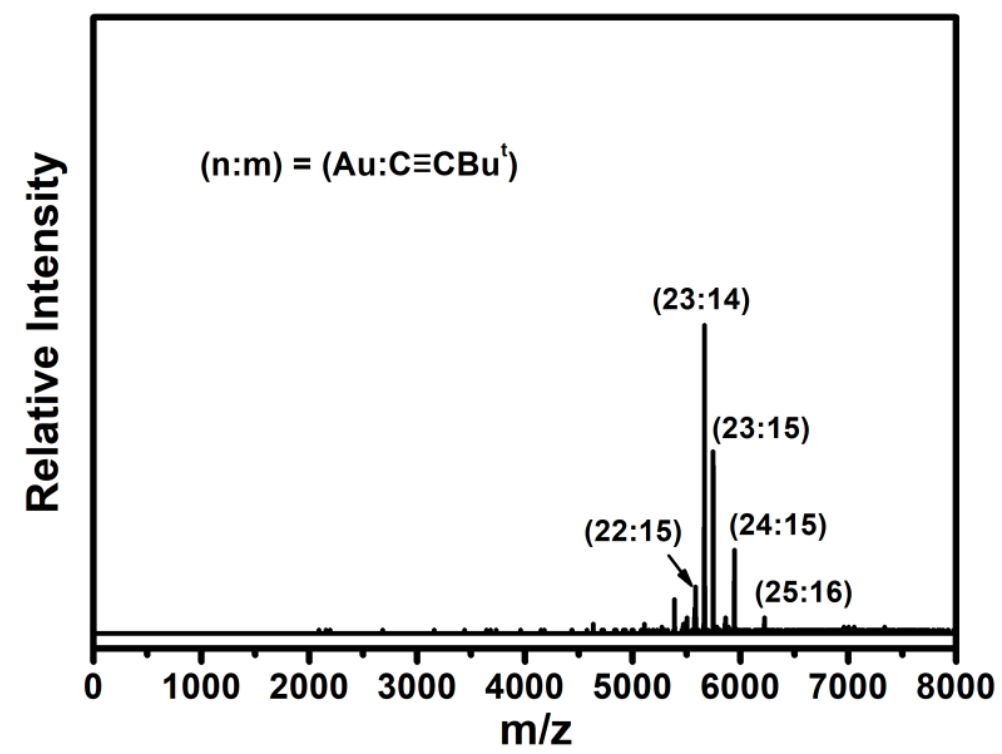

Figure S7. MALDI-TOF mass spectra of the product formed by cluster-conversion reaction of $\mathbf{A u}_{\mathbf{2 3}} \mathbf{- 2}$.

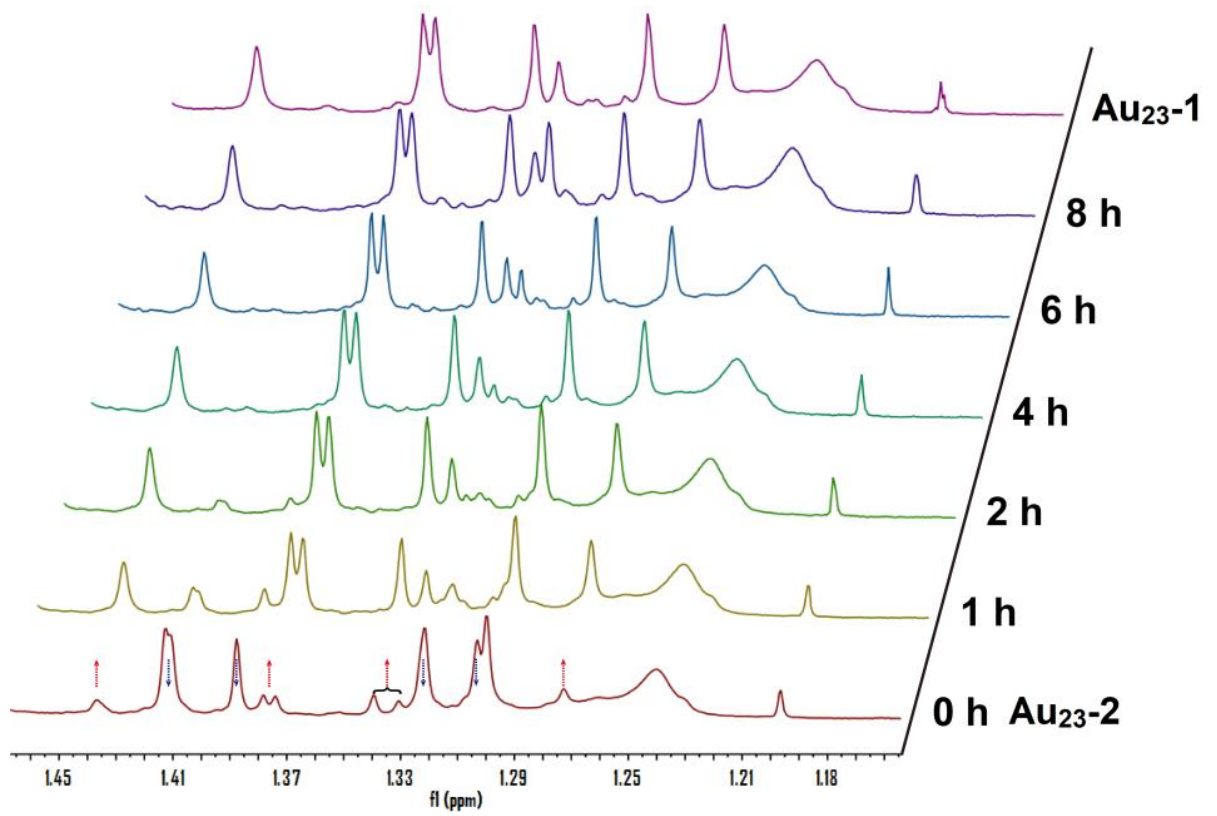

Figure S8. Time-course ${ }^{1} \mathrm{H}$ NMR spectra of cluster-conversion from $\mathbf{A u}_{23}-\mathbf{2}$ to $\mathbf{A u}_{23}-\mathbf{1}$ in $\mathrm{CD}_{2} \mathrm{Cl}_{2}$. 
a)

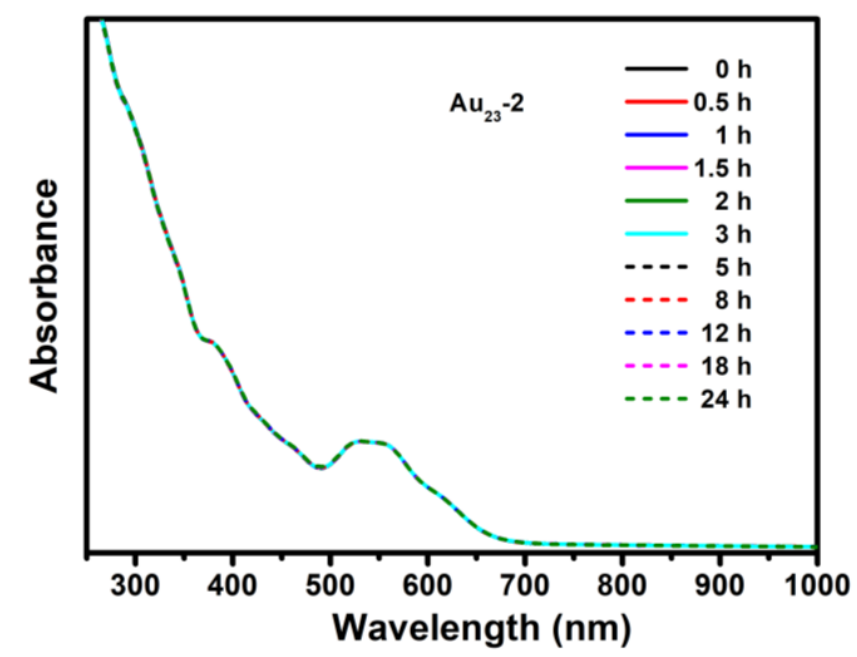

b)

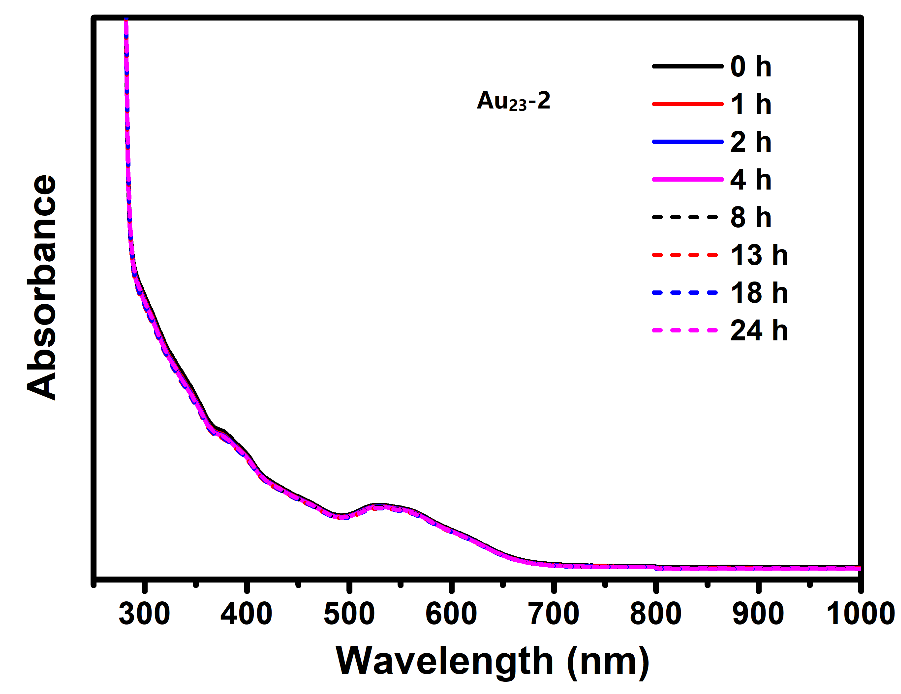

Figure S9. Time-dependent UV-vis absorption spectra of $\mathbf{A u}_{23}-\mathbf{2}$ under different conditions: a) in $\mathrm{CH}_{2} \mathrm{Cl}_{2}$, at $4^{\circ} \mathrm{C}$; b) in $\mathrm{CH}_{2} \mathrm{Cl}_{2}$, at $25^{\circ} \mathrm{C}, 1$ equiv $\mathrm{Ph}_{4} \mathrm{PCl}$ was added.

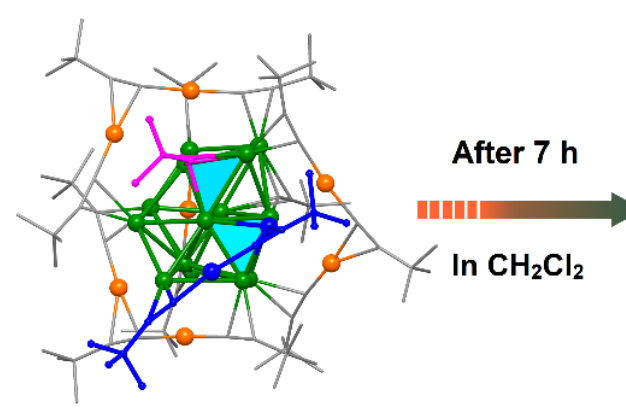

$\mathrm{Au}_{23}-2$

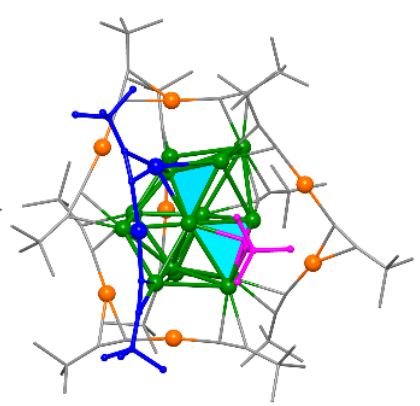

$\mathrm{Au}_{23}-1$

Figure S10. The diagram of transformation process from $\mathbf{A u}_{23}-\mathbf{2}$ to $\mathbf{A u}_{23}-\mathbf{1}$. 


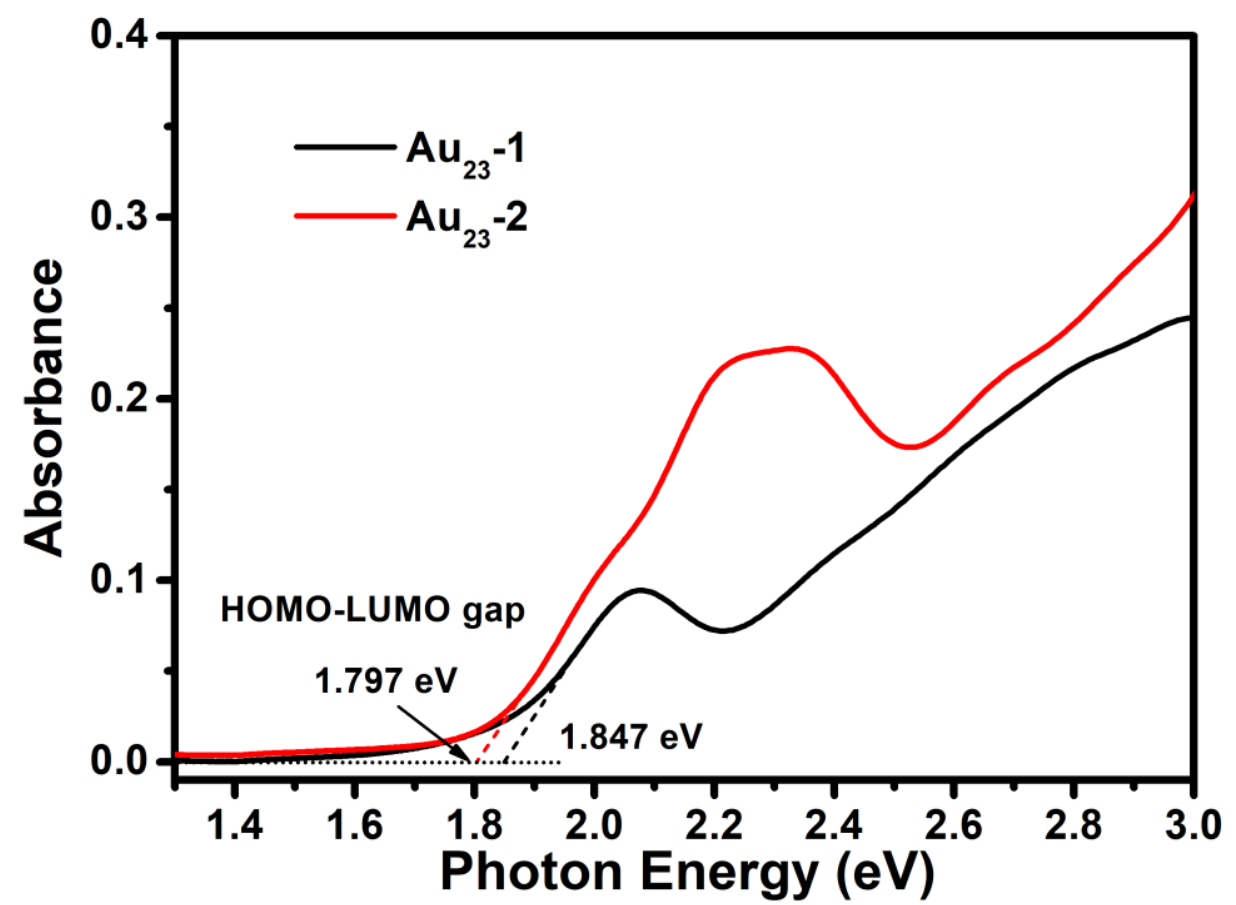

Figure S11. The optical absorption spectra of two $\mathrm{Au}_{23}$ isomers (photon-energy plot).

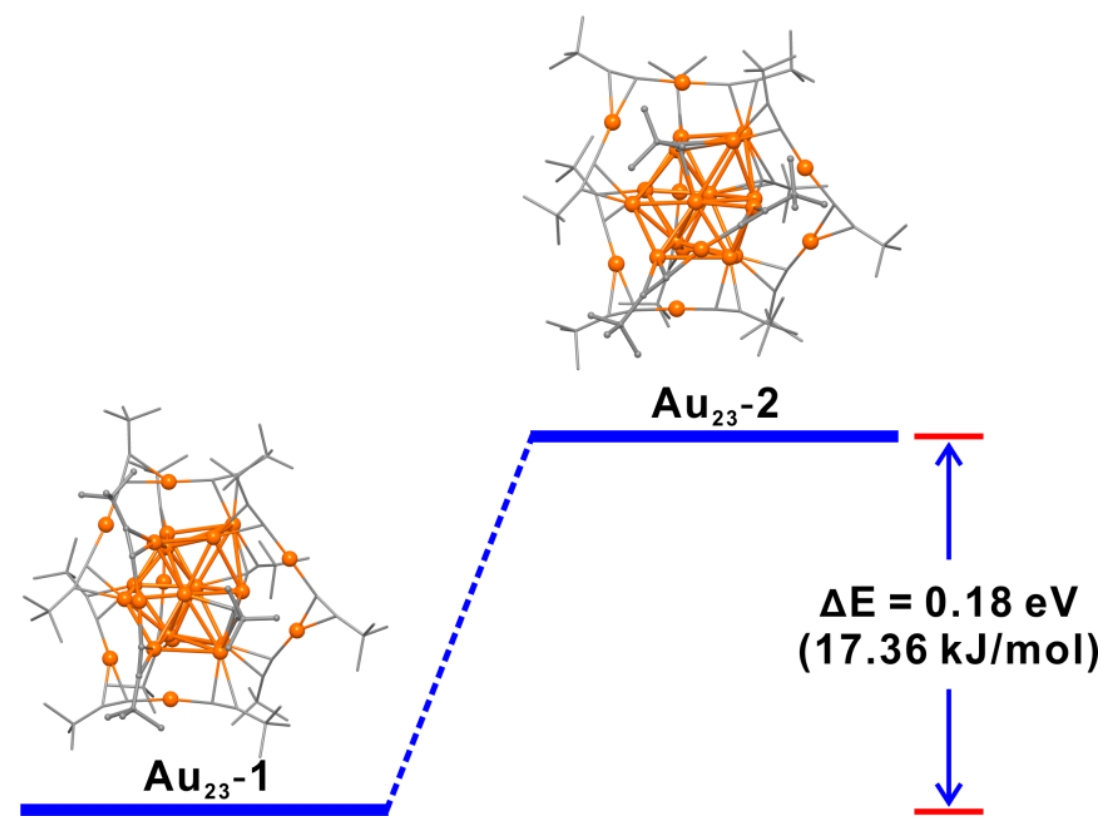

Figure S12. The relative energy difference between $\mathbf{A} \mathbf{u}_{23}-\mathbf{1}$ and $\mathbf{A} \mathbf{u}_{23}-\mathbf{2}$ calculated by Gaussian 09 . 
Table S1. Selected Au-Au bond lengths $(\AA)$ for the Experimental and b3lyp/genecp (6-31G* for C, H and lanl2dz for $\mathrm{Au}$ ) (Unconstrained) optimized structure of $\mathbf{A u}_{\mathbf{2 3}} \mathbf{- 1}$.

\begin{tabular}{|c|c|c|}
\hline Bond & Exp. / ^̊ & Opt / $\AA$ \\
\hline Au9-Au8 & 2.760 & 2.889 \\
\hline Au9-Au5 & 2.786 & 2.884 \\
\hline Au9-Au11 & 2.766 & 2.833 \\
\hline Au9-Au12 & 2.776 & 3.306 \\
\hline $\mathrm{Au} 9-\mathrm{Au} 4$ & 2.706 & 2.810 \\
\hline Au9-Au15 & 2.760 & 2.876 \\
\hline Au8-Au10 & 2.802 & 2.911 \\
\hline Au8-Au7 & 2.738 & 3.299 \\
\hline Au8-Au1 & 2.691 & 2.800 \\
\hline Au8-Au4 & 2.802 & 3.176 \\
\hline Au8-Au15 & 2.669 & 2.897 \\
\hline Au5-Au3 & 2.843 & 3.302 \\
\hline Au5-Au10 & 2.756 & 2.88 \\
\hline Au5-Au13 & 2.681 & 2.899 \\
\hline Au5-Au11 & 2.716 & 2.792 \\
\hline Au5-Au12 & 2.742 & 3.336 \\
\hline $\mathrm{Au} 11-\mathrm{Au} 12$ & 2.727 & 3.255 \\
\hline Au4-Au15 & 2.755 & 3.225 \\
\hline Au10-Au3 & 2.697 & 2.847 \\
\hline Au10-Au2 & 2.872 & 3.020 \\
\hline Au10-Au7 & 2.783 & 3.443 \\
\hline Au10-Au1 & 2.787 & 2.847 \\
\hline Au10-Au13 & 2.767 & 2.833 \\
\hline Au7-Au1 & 2.708 & 3.295 \\
\hline Au3-Au13 & 2.753 & 3.411 \\
\hline Au9-Au2 & 2.896 & 3.042 \\
\hline Au9-Au6 & 2.958 & 2.919 \\
\hline Au8-Au3 & 3.037 & 2.994 \\
\hline Au8-Au6 & 2.922 & 2.919 \\
\hline Au5-Au4 & 3.091 & 3.178 \\
\hline $\mathrm{Au} 11-\mathrm{Au} 4$ & 3.197 & 2.838 \\
\hline $\mathrm{Au} 4-\mathrm{Au} 3$ & 3.028 & 3.083 \\
\hline Au15-Au6 & 3.022 & 2.772 \\
\hline Au10-Au14 & 3.137 & 2.924 \\
\hline $\mathrm{Au} 7-\mathrm{Au} 2$ & 3.008 & 3.44 \\
\hline Au7-Au6 & 2.898 & 3.375 \\
\hline Au1-Au3 & 3.082 & 2.822 \\
\hline Au13-Au14 & 3.074 & 2.786 \\
\hline Au2-Au6 & 2.991 & 3.179 \\
\hline
\end{tabular}


Table S2. Selected Au-Au bond lengths ( $\AA$ ) for the Experimental and b3lyp/genecp (6-31G* for C, H and lanl2dz for $\mathrm{Au}$ ) (Unconstrained) optimized structure of $\mathbf{A u}_{23} \mathbf{- 2}$.

\begin{tabular}{|c|c|c|}
\hline Bond & Exp. / ^̊ & Opt / § \\
\hline $\mathrm{Au} 1-\mathrm{Au} 7$ & 3.155 & 3.381 \\
\hline Au10-Au1 & 2.797 & 2.901 \\
\hline Au10-Au2 & 2.800 & 2.910 \\
\hline Au10-Au7 & 3.063 & 3.254 \\
\hline Au10-Au13 & 2.814 & 2.851 \\
\hline Au10-Au3 & 2.784 & 2.922 \\
\hline Au10-Au23 & 2.685 & 2.909 \\
\hline Au11-Au5 & 2.721 & 2.873 \\
\hline Au11-Au4 & 3.080 & 3.363 \\
\hline Au12-Au23 & 3.050 & 3.089 \\
\hline Au12-Au5 & 2.732 & 2.933 \\
\hline Au12-Au11 & 2.719 & 2.782 \\
\hline Au12-Au9 & 2.751 & 2.888 \\
\hline $\mathrm{Au} 12-\mathrm{Au} 2$ & 3.106 & 3.295 \\
\hline Au13-Au23 & 3.071 & 3.278 \\
\hline Au13-Au3 & 2.714 & 2.778 \\
\hline Au15-Au6 & 3.089 & 3.376 \\
\hline $\mathrm{Au} 2-\mathrm{Au} 1$ & 2.662 & 2.768 \\
\hline $\mathrm{Au} 2-\mathrm{Au} 23$ & 2.822 & 2.970 \\
\hline Au23-Au1 & 2.743 & 2.803 \\
\hline Au4-Au3 & 2.984 & 3.091 \\
\hline $\mathrm{Au} 4-\mathrm{Au} 15$ & 2.758 & 2.814 \\
\hline Au5-Au4 & 3.012 & 3.212 \\
\hline Au5-Au3 & 2.761 & 2.903 \\
\hline Au5-Au13 & 2.693 & 2.883 \\
\hline Au5-Au10 & 2.788 & 2.914 \\
\hline Au5-Au23 & 2.962 & 3.091 \\
\hline Au8-Au6 & 2.982 & 3.349 \\
\hline Au8-Au4 & 2.840 & 2.933 \\
\hline Au8-Au 10 & 2.912 & 2.994 \\
\hline Au8-Au3 & 3.121 & 3.302 \\
\hline Au8-Au 15 & 2.653 & 2.773 \\
\hline $\mathrm{Au} 9-\mathrm{Au} 2$ & 2.946 & 3.022 \\
\hline Au9-Au8 & 2.780 & 2.912 \\
\hline $\mathrm{Au} 9-\mathrm{Au} 4$ & 2.698 & 2.916 \\
\hline Au9-Au5 & 2.791 & 2.897 \\
\hline Au9-Au 15 & 2.773 & 2.877 \\
\hline Au9-Au11 & 2.817 & 2.855 \\
\hline
\end{tabular}


Table S3. The transitions corresponding to the significant peaks of $\mathbf{A u}_{23}-\mathbf{1}$ and $\mathbf{A} \mathbf{u}_{23}-\mathbf{2}$ cluster calculated according to TDDFT method using the quantum chemistry program Gaussian 09.

\begin{tabular}{|c|c|c|c|c|c|c|}
\hline \multirow[t]{8}{*}{ Cluster } & Transition & Coefficient & Contribution $^{\#}$ & $\mathrm{E}(\mathrm{eV}) /(\mathrm{nm})$ & $\begin{array}{l}\text { Oscillator } \\
\text { Strength }\end{array}$ & $\lambda_{\exp }(\mathrm{nm})$ \\
\hline & HOMO -> LUMO & 0.68932 & $95.032 \%$ & $1.70 / 731$ & 0.001 & --- \\
\hline & HOMO-1 -> LUMO & 0.53236 & $56.681 \%$ & $204 / 608$ & 00403 & \multirow{6}{*}{598} \\
\hline & HOMO $\quad$-> LUMO+2 & -0.41864 & $35.052 \%$ & $2.04 / 000$ & 0.0405 & \\
\hline & HOMO $\quad$-> LUMO+1 & 0.56656 & $64.198 \%$ & \multirow{4}{*}{$2.16 / 574$} & \multirow{4}{*}{0.0207} & \\
\hline & HOMO $\quad$-> LUMO+2 & -0.28822 & $16.614 \%$ & & & \\
\hline & HOMO-1 -> LUMO+2 & -0.20153 & $8.123 \%$ & & & \\
\hline & HOMO-1 -> LUMO & -0.15883 & $5.045 \%$ & & & \\
\hline \multirow[t]{5}{*}{$\mathbf{A u}_{23-1}$} & HOMO-2 -> LUMO+2 & 0.43689 & $38.175 \%$ & \multirow{5}{*}{$3.08 / 402$} & \multirow{5}{*}{0.100} & \multirow{5}{*}{412} \\
\hline & HOMO-5 -> LUMO & -0.32290 & $20.853 \%$ & & & \\
\hline & HOMO-3 -> LUMO+1 & 0.26706 & $14.264 \%$ & & & \\
\hline & HOMO $\quad$-> LUMO+4 & 0.14940 & $4.464 \%$ & & & \\
\hline & HOMO-4 -> LUMO & -0.10066 & $2.027 \%$ & & & \\
\hline \multirow{19}{*}{ Aun23-2 } & HOMO $\rightarrow>$ LUMO & 0.60620 & $73.496 \%$ & \multirow{2}{*}{$1.83 / 677$} & \multirow{2}{*}{0.0334} & \multirow{6}{*}{621} \\
\hline & HOMO-1 -> LUMO & 0.32054 & $20.549 \%$ & & & \\
\hline & HOMO-1 -> LUMO & 0.57902 & $67.053 \%$ & \multirow{4}{*}{$1.99 / 622$} & \multirow{4}{*}{0.0591} & \\
\hline & HOMO $\quad>$ LUMO & -0.31753 & $20.165 \%$ & & & \\
\hline & HOMO $\quad$-> LUMO+2 & -0.14540 & $4.228 \%$ & & & \\
\hline & HOMO-1 -> LUMO+1 & -0.13696 & $3.752 \%$ & & & \\
\hline & HOMO $\quad$-> LUMO+2 & 0.47428 & $44.988 \%$ & \multirow{4}{*}{$2.31 / 537$} & \multirow{4}{*}{0.0753} & \multirow{7}{*}{540} \\
\hline & HOMO-1 -> LUMO+1 & -0.40100 & $32.160 \%$ & & & \\
\hline & HOMO-1 $\quad$ > LUMO+2 & 0.23181 & $10.747 \%$ & & & \\
\hline & HOMO $\quad->$ LUMO+2 & 0.14688 & $4.315 \%$ & & & \\
\hline & HOMO-1 -> LUMO+2 & 0.55851 & $62.386 \%$ & \multirow{3}{*}{$2.37 / 524$} & \multirow{3}{*}{0.1038} & \\
\hline & HOMO $\quad$-> LUMO+2 & -0.32567 & $21.212 \%$ & & & \\
\hline & HOMO $\quad$-> LUMO+1 & -0.10656 & $2.271 \%$ & & & \\
\hline & HOMO-2 -> LUMO+2 & 0.42227 & $35.662 \%$ & \multirow{6}{*}{$3.26 / 381$} & \multirow{6}{*}{0.1021} & \multirow{6}{*}{381} \\
\hline & HOMO-4 -> LUMO+2 & 0.21132 & $8.931 \%$ & & & \\
\hline & HOMO $\quad->$ LUMO+5 & -0.20633 & $8.514 \%$ & & & \\
\hline & HOMO-4 -> LUMO+1 & 0.17265 & $5.962 \%$ & & & \\
\hline & HOMO-3 -> LUMO+1 & -0.17131 & $5.869 \%$ & & & \\
\hline & HOMO-6 -> LUMO & 0.16632 & $5.533 \%$ & & & \\
\hline
\end{tabular}


Table S4. Frontier molecular orbital compositions $(\%)$ in the ground state for two $\mathrm{Au}_{23}$ isomers at the CAMB3LYP/6-31G* \& LANL2DZ level.

\begin{tabular}{clcccc}
\hline \multirow{2}{*}{ Cluster } & Orbital & \multicolumn{4}{c}{ Contribution $(\boldsymbol{\%})$} \\
\cline { 3 - 6 } & & Au 15 kernel & Linear staples & V-shaped staples & Bridging alkynyl-ligands \\
\hline \multirow{4}{*}{ Au23-1 } & HOMO-2 & 80.1 & 5.2 & 13.6 & 1.2 \\
& HOMO-1 & 77.4 & 6.2 & 13.4 & 3.1 \\
& HOMO & 74.6 & 5.0 & 17.5 & 2.9 \\
& LUMO & 76.5 & 5.1 & 15.1 & 3.2 \\
& LUMO+1 & 87.5 & 1.0 & 8.2 & 3.3 \\
& LUMO+2 & 83.0 & 0.9 & 13.8 & 2.3 \\
\hline \multirow{5}{*}{ Au23-2 } & HOMO-2 & 88.1 & 3.3 & 8.1 & 0.5 \\
& HOMO-1 & 76.9 & 4.3 & 14.7 & 4.0 \\
& LUMO & 69.4 & 3.9 & 22.8 & 3.9 \\
& LUMO+1 & 81.8 & 1.5 & 12.5 & 3.7 \\
& LUMO+2 & 78.2 & 2.2 & 13.2 & 3.4 \\
\hline
\end{tabular}

Table S5. The transitions corresponding to the significant peaks of $\mathbf{A u}_{\mathbf{2 3}} \mathbf{- 1}$ and $\mathbf{A} \mathbf{u}_{\mathbf{2 3}} \mathbf{- 2}$ cluster calculated according to TDDFT method using Amsterdam Density Functional (ADF 2017) software package.

\begin{tabular}{|c|c|c|c|c|c|c|c|c|}
\hline \multirow{2}{*}{ Cluster } & \multirow{2}{*}{$\begin{array}{c}\triangle \mathrm{E} / \\
\mathrm{eV}\end{array}$} & \multirow{2}{*}{$\begin{array}{c}\text { f (oscillator } \\
\text { strength) }\end{array}$} & \multirow{2}{*}{ Transitions } & \multirow{2}{*}{ Weight } & \multicolumn{3}{|c|}{ Transition dipole moment } & \multirow[t]{2}{*}{$\lambda_{\text {Exp. }} / \mathrm{nm}$} \\
\hline & & & & & $\mathrm{x}$ & $\mathrm{y}$ & $\mathrm{z}$ & \\
\hline \multirow{5}{*}{$\mathbf{A u}_{23-1}$} & 1.4922 & 0.00018 & $\mathrm{HOMO} \rightarrow \mathrm{LUMO}$ & 0.9981 & -0.0227 & -0.0620 & -0.0242 & --- \\
\hline & 1.8318 & 0.03256 & HOMO- $1 \rightarrow$ LUMO & 0.9241 & 1.4652 & 2.9498 & 0.1371 & 598 \\
\hline & 2.2993 & 0.02435 & HOMO-5 $\rightarrow$ LUMO & 0.9397 & -0.2626 & -0.6152 & 0.6478 & 482 \\
\hline & 2.6257 & 0.03652 & $\mathrm{HOMO}-2 \rightarrow \mathrm{LUMO}+2$ & 0.5766 & -0.6297 & -1.1451 & -0.4743 & \multirow{2}{*}{412} \\
\hline & & & HOMO-14 $\rightarrow$ LUMO & 0.1285 & 0.2900 & 0.1335 & 0.1337 & \\
\hline \multirow{11}{*}{ Au23-2 } & 1.8915 & 0.01 & $\mathrm{HOMO} \rightarrow$ LUMO & 0.9063 & 1.7137 & -0.5927 & 1.0076 & \multirow{2}{*}{621} \\
\hline & 1.9974 & 0.02119 & HOMO- $1 \rightarrow$ LUMO & 0.8742 & -1.7447 & 1.0163 & -0.3273 & \\
\hline & \multirow[t]{3}{*}{2.2195} & \multirow[t]{3}{*}{0.03067} & $\mathrm{HOMO} \rightarrow \mathrm{LUMO}+1$ & 0.5483 & 0.7406 & -2.4282 & -1.7095 & \multirow{6}{*}{540} \\
\hline & & & HOMO-2 $\rightarrow$ LUMO & 0.1422 & -0.6709 & 0.6717 & 0.0142 & \\
\hline & & & $\mathrm{HOMO} \rightarrow \mathrm{LUMO}+2$ & 0.1319 & -0.0954 & -0.0533 & -0.3835 & \\
\hline & \multirow[t]{3}{*}{2.3088} & \multirow[t]{3}{*}{0.05382} & HOMO-2 $\rightarrow$ LUMO & 0.6482 & -1.4043 & 1.4059 & 0.0296 & \\
\hline & & & $\mathrm{HOMO} \rightarrow \mathrm{LUMO}+1$ & 0.1165 & -0.3347 & 1.0972 & 0.7724 & \\
\hline & & & HOMO-3 $\rightarrow$ LUMO & 0.1053 & 0.1157 & -0.1642 & 0.2452 & \\
\hline & 2.5530 & 0.03916 & HOMO $-2 \rightarrow$ LUMO+2 & 0.7338 & 0.3381 & 0.6170 & -2.3587 & \multirow{3}{*}{381} \\
\hline & 2.6491 & 0.02109 & HOMO-9 $\rightarrow$ LUMO & 0.6254 & 1.4645 & -0.3604 & 0.5325 & \\
\hline & & & HOMO-10 $\rightarrow$ LUMO & 0.2360 & -0.0922 & 0.6514 & -0.1034 & \\
\hline
\end{tabular}


Table S6. Crystal data and structure refinement for $\mathbf{A u}_{23}-\mathbf{1}$ and $\mathbf{A} \mathbf{u}_{23}-\mathbf{2}$.

\begin{tabular}{|c|c|c|c|c|}
\hline Identification code & \multicolumn{2}{|l|}{$\mathrm{Au}_{23-1}$} & \multicolumn{2}{|l|}{$\mathrm{Au}_{23-2}$} \\
\hline Empirical formula & \multicolumn{2}{|l|}{$\mathrm{C}_{90} \mathrm{H}_{135} \mathrm{Au}_{23}$} & \multicolumn{2}{|l|}{$\mathrm{C}_{90} \mathrm{H}_{135} \mathrm{Au}_{23}$} \\
\hline Formula weight & \multicolumn{2}{|l|}{5747.20} & \multicolumn{2}{|l|}{5747.20} \\
\hline Temperature / K & \multicolumn{2}{|l|}{$100.00(11)$} & \multicolumn{2}{|l|}{100} \\
\hline Crystal system, Space group & \multicolumn{2}{|l|}{ triclinic, $P-1$} & \multicolumn{2}{|l|}{ triclinic, $P-1$} \\
\hline \multirow[t]{3}{*}{ Unit cell dimensions } & $a=16.3376(7) \AA$ & $\alpha=71.615(4)^{\circ}$ & $a=17.3791(5) \AA$ & $\alpha=78.085(3)^{\circ}$ \\
\hline & $b=16.4904(8) \AA$ & $\beta=78.674(4)^{\circ}$ & $b=19.0145(6) \AA$ & $\beta=72.959(3)^{\circ}$ \\
\hline & $c=25.6818(10) \AA$ & $\gamma=60.468(5)^{\circ}$ & $c=19.1440(6) \AA$ & $\gamma=86.481(3)^{\circ}$ \\
\hline Volume / $\AA^{3}$ & \multicolumn{2}{|l|}{$5705.8(5)$} & \multicolumn{2}{|l|}{$5918.2(3)$} \\
\hline$Z, \rho_{\text {calc }} / \mathrm{g} / \mathrm{cm}^{3}$ & \multicolumn{2}{|l|}{$2,3.345$} & \multicolumn{2}{|l|}{$2,3.225$} \\
\hline Absorption coefficient $/ \mathrm{mm}^{-1}$ & \multicolumn{2}{|l|}{29.465} & \multicolumn{2}{|l|}{28.408} \\
\hline $\mathrm{F}(000)$ & \multicolumn{2}{|l|}{4984.0} & \multicolumn{2}{|l|}{4984.0} \\
\hline Crystal size / $\mathrm{mm}^{3}$ & \multicolumn{2}{|l|}{$0.20 \times 0.18 \times 0.03$} & \multicolumn{2}{|l|}{$0.30 \times 0.15 \times 0.12$} \\
\hline Radiation & \multicolumn{2}{|l|}{$\operatorname{MoK} \alpha(\lambda=0.71073)$} & \multicolumn{2}{|l|}{$\operatorname{MoK} \alpha(\lambda=0.71073)$} \\
\hline Theta range for data collection $/{ }^{\circ}$ & \multicolumn{2}{|l|}{6.5 to 50.246} & \multicolumn{2}{|l|}{6.57 to 50.084} \\
\hline Index ranges & \multicolumn{2}{|c|}{$-19 \leq \mathrm{h} \leq 19,-19 \leq \mathrm{k} \leq 19,-29 \leq 1 \leq 30$} & \multicolumn{2}{|c|}{$-20 \leq \mathrm{h} \leq 20,-20 \leq \mathrm{k} \leq 22,-22 \leq 1 \leq 21$} \\
\hline Reflections collected & \multicolumn{2}{|l|}{34127} & \multicolumn{2}{|l|}{39368} \\
\hline Independent reflections & \multicolumn{2}{|c|}{$19497\left[R_{\text {int }}=0.0570, R_{\text {sigma }}=0.0983\right]$} & \multicolumn{2}{|c|}{$20830\left[R_{\mathrm{int}}=0.0710, R_{\mathrm{sigma}}=0.1299\right]$} \\
\hline Data / restraints / parameters & \multicolumn{2}{|l|}{19497 / 534 / 1090} & \multicolumn{2}{|l|}{$20830 / 582 / 1081$} \\
\hline Goodness-of-fit on $F^{2}$ & \multicolumn{2}{|l|}{1.037} & \multicolumn{2}{|l|}{1.048} \\
\hline Final $R$ indexes $[I>=2 \sigma(I)]$ & $R_{1}=0.0608, w R_{2}=0$ & 359 & $R_{1}=0.0701, w R_{2}=$ & 1521 \\
\hline Final $R$ indexes [all data] & $R_{1}=0.0815, w R_{2}=0$ & 490 & $R_{1}=0.0988, w R_{2}=$ & 1717 \\
\hline Largest diff. peak / hole / e. $\AA^{-3}$ & $3.99 /-3.52$ & & $4.60 /-4.51$ & \\
\hline
\end{tabular}

\section{References}

1. Lee, D.; Donkers, R. L.; Wang, G.; Harper, A. S.; Murray, R. W. J. Am. Chem. Soc. 2004, 126, 6193-6199.

2. Kang, X.; Wang, S.; Song, Y.; Jin, S.; Sun, G.; Yu, H.; Zhu, M. Angew. Chem. Int. Ed. 2016, 55, 3611-3614.

3. Kang, X.; Huang, L.; Liu, W.; Xiong, L.; Pei, Y.; Sun, Z.; Wang, S.; Wei, S.; Zhu, M. Chem. Sci. 2019, 10, 86858693.

4. Gaussian 09, Revision A.1, M. J. Frisch, G. W. Trucks, H. B. Schlegel, G. E. Scuseria, M. A. Robb, J. R. Cheeseman, G. Scalmani, V. Barone, B. Mennucci, G. A. Petersson, H. Nakatsuji, M. Caricato, X. Li, H. P. Hratchian, A. F. Izmaylov, J. Bloino, G. Zheng, J. L. Sonnenberg, M. Hada, M. Ehara, K. Toyota, R. Fukuda, J. Hasegawa, M. Ishida, T. Nakajima, Y. Honda, O. Kitao, H. Nakai, T. Vreven, J. A. Montgomery, Jr., J. E. Peralta, F. Ogliaro, M. Bearpark, J. J. Heyd, E. Brothers, K. N. Kudin, V. N. Staroverov, R. Kobayashi, J. Normand, K. Raghavachari, A. Rendell, J. C. Burant, S. S. Iyengar, J. Tomasi, M. Cossi, N. Rega, J. M. Millam, M. Klene, J. E. Knox, J. B. Cross, V. Bakken, C. Adamo, J. Jaramillo, R. Gomperts, R. E. Stratmann, O. Yazyev, A. J. Austin, R. Cammi, C. Pomelli, J. W. Ochterski, R. L. Martin, K. Morokuma, V. G. Zakrzewski, G. A. Voth, P. Salvador, J. J. Dannenberg, S. Dapprich, A. D. Daniels, Ö . Farkas, J. B. Foresman, J. V. Ortiz, J. Cioslowski, and D. J. Fox, Gaussian, Inc., Wallingford CT, 2009M.

5. Bomble, Y. J. J. Am. Chem. Soc. 2006, 128, 3103-3103.

6. Becke, A. D. Phys. Rev. A. 1988, 38, 3098-3100.

7. Hay, P. J.; Wadt, W. R. J. Chem. Phys. 1985, 82, 299-310.

8. Yanai, T.; Tew, D. P.; Handy, N. C. Chem. Phys. Lett. 2004, 393, 51-57.

9. Gritsenko O. V.; Schipper P. R. T.; Baerends E. J. Chem. Phys. Lett. 1999, 302, $199-207$. 